\title{
Proactive Therapy and Emollient Therapy in Atopic Dermatitis
}

\author{
A. P. Oranje, PhD $M D^{1 * 2,3}$ \\ Address \\ ${ }^{1 *}$ Maasstad Hospital, Rotterdam, The Netherlands \\ Email: a.oranje@inter.nl.net \\ ${ }^{2}$ Dermicis Skin Hospital, Alkmaar, The Netherlands \\ ${ }^{3}$ Intermedica Hair Clinic, Boxmeer, The Netherlands
}

Published online: 17 0ctober 2014

(C) Springer International Publishing AG 2014

Keywords Atopic dermatitis - Proactive treatment - Emollients · Crisis intervention • Long-term intervention

Abbreviations $A D$ atopic dermatitis or eczema - WWT wet-wrap treatment

\section{Opinion statement}

Atopic dermatitis is a common itching disorder that includes the majority of my work in the university clinic of the KinderHaven, Havenziekenhuis/Erasmus MC, Rotterdam. Treatment was always chosen on the basis of severity established with the scoring system, 'objective SCORAD'. Follow-up may be monitored with a simplified (Target) scoring system called the Three-items-severity Score (TIS), that was developed into the late 1990s in Holland and in particular in Rotterdam by Glazenburg and Oranje. Recently, the HOME group proposed to accept only the EASI as the scoring system and to no longer to use the others. This is totally in disagreement with the author's opinion and many others of the European Task Force for Atopic Dermatitis. This recommendation of HOME is based on subjective assessment of a group that just partly representing the scientific community. Proactive topical therapy is preferred above other topical treatment schedules. It is a variation of the step-up/step-down strategy (Van der Meer, Glazenburg, Oranje) that starts with intensive and aggressive topical anti-inflammatory therapy until control is achieved. Proactive treatment is defined as "long-term, low dose intermittent topical anti-inflammatory therapy for previously affected areas with subclinical inflammation and involves a behavioral concept" (Wollenberg A, J Dtsch Dermatol Ges 2009;7:117-21) [1]. Both visible and non-visible eczema is treated with at least twice weekly application. Aggressive active treatment is started again in case of recurrence. Emollients have become increasing important in the treatment of atopic dermatitis. In earlier years, there were simply prepared creams or ointments. However, today there are more sophisticated creams and ointments available in an attempt to repair the skin barrier function in atopic dermatitis. It is better to refer to these as moisturizers. Future emollients or moisturizers are expected to have specific properties to restore the 
barrier function even more efficiently in atopic dermatitis. This manuscript is based on the expert opinion of the author.

\section{Introduction}

Atopic dermatitis (with a synonym of atopic eczema or 'eczema') is a common itching dermatitis with a strong genetic basis combined with and influenced in severity by a multifactorial background. Atopic dermatitis (AD) is characterized by exacerbations and remissions and need a multifaceted treatment strategy [2•].

Together with food allergies, asthma, and allergic rhinitis, AD belongs to the Atopy Syndrome. The Atopic March (the follow-up of signs and symptoms) explains the time-line of the different expressions of the Atopy syndrome.

Treatment is started based on the severity of $\mathrm{AD}$. Systemic therapy is necessary in only $10 \%$ of particularly severe and recalcitrant $\mathrm{AD}$. Scoring atopic dermatitis with the (SCORAD)-index, the objective SCORAD, and the EASI are the most validated systems for evaluating the severity. However, it seems that the HOME group prefers the EASI above the others (unpublished observations). The people voting at HOME were some methodologists, people from industry, patients, and others, but only a few expert physicians. Many members of the scientific community disagree with their recommendations.

Follow-up may be evaluated by the target or the global scoring of the scratches, the oedema, and the erythema, the so-called Three-item Severity Score (TIS) [3, 4].
Some dermatologists have practised an alternative approach in the last 20 years in which they almost never stop with the treatment, but then continue with the application of the creams or the ointments only twice weekly also on sites where eczema was previously treated with a combined application of emollients or vehicles of the active drugs. At the end of the 1990s it was referred to as 'step-down' therapy by Glazenburg, Van der Meer and Oranje [5, 6]. In these studies, a target screening test for severity was introduced, later named TIS by Wolkerstorfer and Oranje [3]. Follow-up of the patients may be done with an easier system such as the TIS evaluation.

Wet-wrap treatment (WWT) for severe retractable AD was also recently described as a proactive manner for long-term treatment [7•]. Dry occlusive therapy was reported to have no additional benefit above the conventional open therapy [8]. Although WWT is often practised with diluted corticosteroids and emollients or emollients alone, there is limited evidence of their efficacy [5].

Both the general and the specific aspects of topical management concerning the proactive treatment and the use of emollients in $\mathrm{AD}$ are described in this review article.

\section{Therapy: general aspects}

Current therapy of $\mathrm{AD}$ is directed at suppressing the inflammation and reducing the triggering factors. Goals of the current standard therapy are an improvement in the skin-barrier function, the blocking of receptors and a reduction of the inflammatory infiltrate. Advanced treatment of severe AD in children includes:

- General and preventive aspects

- An individualised therapeutic regimen and the use of emollients

- Topical application of corticosteroids or application of topical immune modulators

- Wet-wrap techniques using diluted corticosteroids or antiseptics

- Topical or systemic antibiotics

- Systemic treatment in recalcitrant disease (cyclosporine, azathioprine) 
- Dietary elimination and oral probiotics

- Other and experimental therapies

Most patients with atopic dermatitis respond to a regimen that consists of bleach baths at least 2-3 times weekly, eventual antibiotic ointment for the nose, emollients, and topical steroids [9, 10]. More severe cases may require allergen avoidance and more intense education and psychological support for the family. Therefore, written instruction plans and eczema school settings are important tools. A "fourth generation" of topical steroids, including both mometasone and fluticasone, appear to have an improved therapeutic index with less adverse effects noted after use for more than two weeks.

In erythrodermic or severe refractory $\mathrm{AD}$, different variations of wet-wrap techniques may be used. The wet-wrap technique is a sort of occlusive treatment. The resorption of the topical corticosteroid is increased to $10 \%$ under occlusion, while hydration of the skin leads to a fourfold to fivefold increase in absorption [11]. This therapy with a potent diluted corticosteroid cream with minor systemic adverse effects that is not only an interesting and promising option in children but also in adults. The WWT is usually more effective than treatment with the topical immunomodulators (TIM), including tacrolimus and pimecrolimus creams or ointments. Both of these immunomodulatory drugs have demonstrated efficacy when used in the treatment of moderate and severe atopic dermatitis. Toxicity has thus far been minimal with the use of either agent.

Other therapies that have been used in severe disease include phototherapy (in children preferably with UVB) and systemic therapy such as cyclosporine, methotrexate, and azathioprine. There is mostly evidence for systemic cyclosporine therapy. Cyclosporine is typically used as crisis intervention for three months and patients must be carefully followed for renal toxicity. Rare cases of markedly increased alkaline phosphatase have also been reported [12]. Relapse usually occurs shortly after discontinuation of therapy. Systemic therapy will not be dealt with in this article.

There is no evidence-based information about the positive or negative effects of bathing, and in which way it should be performed. In the Dutch Guidelines for atopic eczema it is stated that you should bath in lukewarm water, for a short time, and three times weekly. (NHG standard text, 2014).

I do recommend daily bathing (maximum time of five minutes), application of emollients, and use of soap substitutes (so-called syndets).

Antiseptic baths at least twice weekly are often recommended to diminish the number and severity of skin infections. A problem is that this treatment is also destructive for so-called good bacteria. An old remedy is to use Potassium Permanganate (KMnO4) in the bath, but the disadvantage is the staining. Literature is sparse, but from an old article dated 1964 by Brit, I cite:

"Potassium permanganate is the simplest agent for the use in the control of secondary skin infections in eczema. Through oxidation it controls bacteria and it also dries denuded areas. Potassium permanganate should be prepared freshly for each bath, in a right dilution of 1:6o0" [13]. An important disadvantage is the pink-brown staining of towels, bath-tubs, and other material. 


\section{Emollients and atopic dermatitis}

Diluted bleach baths have been investigated as extremely useful and effective by the group of Amy Paller [10].

Huang published the first study on the use of diluted bleach baths in patients with overt secondary skin infection in atopic eczema [14]. Diluted bleach baths with intermittent intranasal application of mupirocin ointment decreased the clinical severity of atopic eczema in patients with clinical secondary skin infections $[10,14]$.

Skin dryness is a highly important aspect of AD. The skin barrier function is impaired allowing an easy penetration of bacteria, other microorganisms, allergens, and toxic substances into the skin.

Hydration of the skin can be improved by at least twice daily or even more applications of emollients (up to six times daily) with a hydrophilic base completely indifferent or containing approximately a maximum of $5 \%$ urea and/or lactic acid, if tolerated. Emollients can be defined as indifferent moisturizers with limited adding to the cream or the ointment. Today, more and more moisturizers have become available with different components added in an attempt to repair the disrupted skin- barrier function. It is difficult to distinguish these products from active topical drugs.

At least there is some evidence-based support that emollients will help to reduce the dryness of the skin, thereby improving the barrier function and improving eczema. It was also reported in different studies that emollients do have a corticosteroid- sparing effect [14-16]. The following was stated in a recent review: "Some over-the-counter (OTC) ingredients such as colloidal oatmeal

and petrolatum, as well as anti-inflammatory prescription moisturizers (medical device creams), have demonstrated efficacy with little complications in skin barrier repair and symptom relief in steroid-responsive conditions. With regimented application, these non-drug options are safe and effective and can limit the long-term continuous use of topical corticosteroids or topical calcineurin inhibitors [17•]."

Direct topical use of emollients or moisturizers on actively inflamed skin is poorly tolerated in $\mathrm{AD}$. Flares of $\mathrm{AD}$ need to be treated with wet dressings, topical corticosteroids or topical calcineurin inhibitors [18•].

\section{Step-down (step-up) therapy in the maintenance phase}

Different regimens for the use of corticosteroids were introduced for the long-term treatment and optimal control of $\mathrm{AD}$ in the 1990s. One such regimen was called the step-down therapy. At the start of the treatment, a potent steroid is applied for two weeks or even only three days, then a weaker steroid is applied to maintain the effect of the therapy. An alternative that I used from the 1990s, is the application of a potent steroid daily, after two weeks four days per week, and then after four weeks again attempt to reduce the frequency of application for maintenance of the therapy effect. This treatment is currently still the approach used at our clinic as also in WWT [4]. Several studies with fluticasone propionate were performed in this manner $[4,5]$. When eczema 
exacerbated, therapy returned again to the active aggressive mode and then tapered off again according to the protocol followed.

Proactive therapy

\section{Education of the parents}

Proactive therapy as a term and a concept was introduced in 2009 by Wollenberg and Bieber [19].

This is an alternative treatment that includes an intensive topical antiinflammatory therapy until all lesions have mostly cleared, followed by longterm, low dose intermittent application of anti-inflammatory therapy to the former affected areas combined with daily applications of emollients to unaffected areas. That is completely different from reactive treatment in which the treatment consists of daily application of emollients, and, if necessary, antiseptics and topical anti-inflammatory drugs on " needed basis " [19].

However, proactive therapy is in reality basically a little bit different from the step-down therapy described earlier. The first study presenting step-down therapy was by Van der Meer [4]. 'Proactive step-down' therapy also starts with intensive and aggressive topical anti-inflammatory therapy until control is achieved. Thereafter, one can continue with maintenance treatment using different schedules. Both visible and non-visible eczema are treated with at least twice to four times weekly application. However, this is not always possible in every patient, when you may need three or four weekly applications. The success of this approach was reported in many studies $[5,6,7 \bullet, 20-25]$.

Treatment with a proactive strategy demands much discipline from the parents and the patients. It means that you have to spend much time to train the parents: how to cope with pruritus, how to apply the creams or the ointments, and not to stop the treatment early.

There are four ways to manage this:

1. Teaching the parents and the patients by offering extra attention via an eczema school project.

2. In-between explanation of proactive treatment, guidance and help from a nurse-practitioner specialized in the care of eczema.

3. Offering in-between email contacts to answer questions and support the parents.

4. Offering hand outs with written instructions.

Active participation of the parents should be encouraged and is essential in the management of care and more successful treatment. It is also essential to accept that AD cannot be cleared completely [26•].

\section{Discussion}

We learnt from our teachers that you should be careful with potent corticosteroids by treating patients in as short as possible time in a reactive manner. However, proactive treatment and step-down treatment changed this concept completely, as 
shown in several studies. A recent review on all those concepts of induction of remission and treatment of a sub-clinical or non-visible eczema showed that in 20 of the 26 included studies, there was evidence of a sub-clinical inflammation with a change from normal-appearing skin or post-treatment lesion skin to active skin lesions in patients with AD. Such sub-clinical inflammation was reported tp improve with proactive treatment aimed at maintaining remission. Failure to achieve control of $\mathrm{AD}$ symptoms with initial therapy was associated with a higher risk of relapse in 14 randomized controlled trials both with fluticasone propionate cream or ointment and with tacrolimus ointment [27•].

Proactive treatment showed better results than reactive treatment in which only a small number of patients experienced a long-term remission of eczema. This is also the opinion of the author [1].

Topical corticosteroids or topical calcineurin inhibitors are necessary to suppress and control $\mathrm{AD}$, especially as flares only respond adequately to these anti-inflammatory drugs. For maintenance and to prevent recurrences, emollients and moisturizers are highly important because of their corticosteroidsparing or calcineurin inhibitor- sparing effects. Today, emollients are more sophisticated and attempt to restore the epidermal barrier dysfunction and to supplement or alter the shortcomings in the epidermis. Genetic background, immune dysregulation and abnormal proteins in the skin are responsible for this epidermal barrier dysfunction [14].

Proactive treatment, emollient supplementation and bleach baths in cases of overt secondary infection are the key steps now of the modern treatment of $\mathrm{AD}$ $[1,13,16]$.

Emollients are widely used and inexpensive in most cases, but there are ever increasing dangers of contact sensitization because of their complicated composition.

Contact sensitization or irritation by components of the emollients/ moisturizers may worsen the skin condition in $\mathrm{AD}$ and influence the course and the severity of eczema, especially by the use of complex composed moisturizers. Moreover, sensitized atopic subjects may respond to very low concentrations of contact allergens because of their impaired skin barrier function and hyper-reactivity to irritant stimuli enhancing contact reactions.

A positive effect of combining proactive treatment with emollients may be that emollients limit the use of therapies such as topical corticosteroid creams/ ointments and calcineurin inhibitor cream or ointments to when they are actually needed. It is also still unclear which emollients are the most effective and whether it is important to have a choice of different emollients for different body sites. We also need to know how emollients should be used and with what frequency (usually twice daily is recommended). This is currently completely unknown. I advise parents to try to apply emollients 2-6 times daily.

The skin of AD patients is highly colonized by Staphylococcus aureus and other staphylococcal species persisting in areas of dry skin as well in mildly affected skin. The current hypothesis is that staphyloccoccal exotoxins are thought to act as super-antigens causing exacerbations of eczema. Therefore, increased staphylococci colonization/infection may be responsible for flares. Reducing bacterial load will result in clinical improvement.

The human cutaneous microbiome is an ecosystem composed of trillions of microorganisms (bacteria, yeast, viruses, fungi, etc.), which colonise the stratum corneum. Recently, it was reported that the microbiome or 
biofilm in AD differs from that in normal skin. Staphylococci form a biofilm by playing a dominant role in the occlusion of sweat ducts, leading to inflammation and pruritus [28•].

This may also explain why in recurrent secondary infected eczema regular baths of diluted bleach (sodium hypochlorite) and intranasal application of mupirocin ointment reduces the severity of $\mathrm{AD}$ in children as well as in adults. Severe childhood AD was reported to be successfully treated with wet- wraps ('wet pyjama") and not only with diluted corticosteroids, but also with antiseptics illustrating once again the importance of the biofilm in AD. Possible future developments of moisturizers will include products with antibacterial activities that will restore a balanced microbiome in the damaged skin in AD.

\section{Compliance with Ethics Guidelines}

\section{Conflict of Interest}

Arnold P. Oranje declares that he has no conflict of interest.

\section{Human and Animal Rights and Informed Consent}

This article does not contain any studies with human or animal subjects performed by any of the authors.

\section{References and Recommended Reading}

Papers of particular interest, published recently, have been highlighted as:

- Of importance

1. Wollenberg A, Frank R, Kroth J, Ruzicka T. Proactive therapy of atopic eczema-an evidence-based concept with a behavioral background. J Dtsch Dermatol Ges. 2009; 7:117-21.

2. $\quad$ Oranje AP. Evidence - based pharmacological treatment of atopic dermatitis: an expert opinion and new expectations. Indian J Dermatol. 2014;59:140-2.

Systemic treatment of AD is discussed with regards to the evidence-based efficacy and safety aspects, and only for Cyclosporine is enough evidence is found. Wet-wraps with diluted corticosteroids are excellent as a crisis intervention in severe childhood cases. Probiotics are not useful in the treatment. Anti-histamines are not effective against pruritus in AD. They are only effective against urticarial flares and in cases with a food allergy

3. Oranje AP, Glazenburg EJ, Wolkerstorfer A, de Waardvan der Spek FB. Practical issues on interpretation of scoring atopic dermatitis: the SCORAD index, objective SCORAD and the three-item severity score. $\mathrm{Br} \mathrm{J}$

Dermatol. 2007;157:645-8.
4. Wolkerstorfer A, de Waard van der Spek FB, Glazenburg EJ, Mulder PG, Oranje AP. Scoring the severity of atopic dermatitis: three item severity score as a rough system for daily practice and as a pre-screening tool for studies. Acta Derm Venereol. 1999;79:356-9.

5. Van Der Meer JB, Glazenburg EJ, Mulder PG, Eggink HF, Coenraads PJ. The management of moderate to severe atopic dermatitis in adults with topical fluticasone propionate. The Netherlands Adult Atopic Dermatitis Study Group. Br J Dermatol. 1999;140:1114-21.

6. Glazenburg EJ, Wolkerstorfer A, Gerretsen AL, Mulder PG, Oranje AP. Efficacy and safety of fluticasone propionate $0.005 \%$ ointment in the long-term maintenance treatment of children with atopic dermatitis: differences between boys and girls? Pediatr Allergy Immunol. 2009;20:59-66.

7. $\quad$ Janmohamed SR, Oranje AP, Devillers AC, et al. The proactive wet-wrap method with diluted corticosteroids versus emollients in children with atopic 
dermatitis: A prospective, randomized, double-blind, placebo-controlled trial. J Am Acad Dermatol.

2014;70:1076-82.

Wet wraps with diluted corticosteroids for severe AD is an effective therapy option for at least a period of four weeks. For the longer term, the proactive approach with WWT is very useful and safe if supervised by a nurse-practitioner

8. Braham SJ, Pugashetti R, Koo J, et al. Occlusive therapy in atopic dermatitis: overview. J Dermatol Treat. 2010;21:62-72.

9. Fritz SA, Camins BC, Eisenstein KA, Fritz JM, Epplin EK, Burnham CA, et al. Effectiveness of measures to eradicate Staphylococcus aureus carriage in patients with community-associated skin and soft-tissue infections: a randomized trial. Infect Control Hosp Epidemiol. 2011;32:872-80.

10. Paller AS, Simpson EL, Eichenfield LF, Ellis CN, Mancini AJ. Treatment strategies for atopic dermatitis: optimizing the available therapeutic options. Semin Cutan Med Surg. 2012;31(3

Suppl):S10-7.

11. Oranje AP, Devillers AC, Kunz B, Jones SL, DeRaeve L, Van Gysel D, et al. Treatment of patients with atopic dermatitis using wet-wrap dressings with diluted steroids and/or emollients. An expert panel's opinion and review of the literature. J Eur Acad Dermatol Venereol. 2006;20:1277-86.

12. van Meurs T, Wolkerstorfer A, Oranje AP. Extreme rises in serum alkaline phosphatase in children with atopic dermatitis after intervention treatment with cyclosporin A. Pediatr Dermatol. 1998;15:483.

13. Birt AR. Drugs for eczema of children. Can Med Assoc J. 1964;90:693-4.

14. Huang JT, Abrams M, Tlougan B, Rademaker A, Paller AS. Treatment of Staphylococcus aureus colonization in atopic dermatitis decreases disease severity. Pediatrics. 2009; 123:e808-14.

15. Grimalt R, Mengeaud V, Cambazard F, Study Investigators' Group. The steroid-sparing effect of an emollient therapy in infants with atopic dermatitis: a randomized controlled study. Dermatology.

2007;214:61-7.

16. Msika P, De Belilovsky C, Piccardi N, Chebassier N, Baudouin C, Chadoutaud B. New emollient with topical corticosteroid-sparing effect in treatment of childhood atopic dermatitis: SCORAD and quality of life improvement. Pediatr Dermatol. 2008;25:606-12.

17. Harcharik S, Emer J. Steroid-sparing properties of emollients in dermatology. Skin Therapy Lett. 2014;19:5-10.

Some over-the-counter (OTC) ingredients such as colloidal oatmeal and petrolatum, as well as anti-inflammatory prescription moisturizers (medical device creams), have demonstrated efficacy with little complications in skin barrier repair and symptom relief in steroid-responsive conditions. With regimented application, these non-drug options are safe and effective and can limit the long-term continuous use of TCS or TCIs
18. Gelmetti C, Wollenberg A. Atopic dermatitis - all you can do from the outside. Br J Dermatol. 2014;170 Suppl 1:19-24.

This contribution outlines a number of strategies for effectively managing AD, from reactive therapy using TCS and TCI to proactive therapy. Proactive therapy is an alternative, evidencebased, immunologically founded treatment approach, based on the fact that normal looking, non-lesional skin of patients with $\mathrm{AD}$ is not normal. The advantage of the proactive approach is that the patients are in control of their disease and are actively involved in its management. The avoidance of external irritants is recommended wherever possible

19. Wollenberg A, Bieber T. Proactive therapy of atopic dermatitis-an emerging concept. Allergy.

2009;64:276-8.

20. Darsow U, Wollenberg A, Simon D, Taïeb A, Werfel T, Oranje A, et al. Difficult to control atopic dermatitis. World Allergy Organ J. 2013;6:1-6.

21. Berth-Jones J, Damstra RJ, Golsch S, Livden JK, Van Hooteghem O, Allegra F, et al. Twice weekly fluticasone propionate added to emollient maintenance treatment to reduce risk of relapse in atopic dermatitis: randomised, double blind, parallel group study. BMJ. 2003;326:1367.

22. Hanifin J, Gupta AK, Rajagopalan R. Intermittent dosing of fluticasone propionate cream for reducing the risk of relapse in atopic dermatitis patients. $\mathrm{Br} J$ Dermatol. 2002;147:528-37.

23. Breneman D, Fleischer Jr AB, Abramovits W, Zeichner J, Gold MH, Kirsner RS, et al. Tacrolimus Ointment Study Group. Intermittent therapy for flare prevention and long-term disease control in stabilized atopic dermatitis: a randomized comparison of 3-times-weekly applications of tacrolimus ointment versus vehicle. J Am Acad Dermatol. 2008;58:990-9.

24. Thaçi D, Reitamo S, Gonzalez Ensenat MA, Moss C, Boccaletti V, Cainelli T, et al. European Tacrolimus Ointment Study Group. Proactive disease management with $0.03 \%$ tacrolimus ointment for children with atopic dermatitis: results of a randomized, multicentre, comparative study. Br J Dermatol. 2008;159:1348-56.

25. Wollenberg A, Ehmann LM. Long term treatment concepts and proactive therapy for atopic eczema. Ann Dermatol. 2012;24:253-60.

26. Barbarot S, Stalder JF. Therapeutic patient education in atopic eczema. Br J Dermatol. 2014;170 Suppl 1:44-8. Involvement of patient associations in the Therapeutic Patient Education (TPE) process varied from one country to another. Despite the nonexhaustive approach, our findings demonstrate the increasing interest in TPE for managing individuals with $\mathrm{AD}$. In spite of the cultural and financial differences between countries, there is a consensus among experts to integrate education into the treatment of eczema

27. Tang TS, Bieber T, Williams HC. Are the concepts of induction of remission and treatment of subclinical inflammation in atopic dermatitis clinically useful? J Allergy Clin Immunol. 2014;133:1615-25. Induction of remission followed by maintenance therapy might 
prove to be an integral part of a disease-modifying strategy for treating atopic dermatitis.

Induction of remission followed by maintenance therapy might prove to be an integral part of a disease-modifying strategy for treating atopic dermatitis

28.• Allen HB, Vaze ND, Choi C, Hailu T, Tulbert BH, Cusack CA, et al. The presence and impact of biofilm- producing staphylococci in atopic dermatitis. JAMA Dermatol. 2014;150:260-5.

Biofilm formation by AD-associated staphylococci almost certainly plays a major role in the occlusion of sweat ducts. Biofilm formation leads to inflammation and pruritus. The environmental hit in AD relates to staphylococci and their biofilms, which occlude sweat ducts 